\title{
LA PERCEPCIÓN DEL CONSUMIDOR SOBRE EL PRODUCTO TURÍSTICO: EL CASO DE TABASCO.
}

\author{
Andrés Guzmán Sala ${ }^{1}$, Armando Mayo Castro², Beatríz Pérez Sánchez ${ }^{3}$
}

\begin{abstract}
Guzmán-Sala A., Mayo-Castro A., Pérez-Sánchez B. La percepción del consumidor sobre el producto turístico: El caso de Tabasco. Hitos de Ciencias Económico Administrativas 2012;18 (51):55-62.
\end{abstract}

RESUMEN

Objetivo. Estudiar el producto turístico enfocado hacia los establecimientos hoteleros, restauranteros, y transporte turístico, como componentes importantes de la oferta turística en Tabasco, desde la perspectiva del turista, con la finalidad de revelar el estado de sus atributos, respecto al servicio, precio y promoción.

Materiales y métodos. Investigación cuantitativa, transversal, descriptiva del producto turístico en Tabasco, con datos recopilados del Instituto Nacional de Estadística y Geografía y la Secretaría de Turismo, incluyendo aportaciones de importantes autores en economía turística. Se aplicaron 271 cuestionarios a los turistas, en base a la muestra de la población, con un nivel de confianza del $90 \%$. Se obtuvieron datos sobre el servicio proporcionado en los sectores hotelero, restaurantero, y en el transporte turístico. Tres variables importantes fueron estudiadas: producto, precio y promoción.

Resultados. En los tres sectores, los servicios son percibidos como buenos por los turistas, en donde los porcentajes más elevados por arriba del $60 \%$ se observaron en las habitaciones de hotel y la alimentación en restaurantes. Los precios presentan un comportamiento percibido como bueno en los 3 sectores turísticos, aclarando que solo en habitación de hotel se observa un porcentaje arriba del $60 \%$. Pero
Guzmán-Sala A., Mayo-Castro A., Pérez-Sánchez B. The consumer perception on tourist product: the case of Tabasco. Hitos de Ciencias Económico Administrativas 2012;18 (51):55-62.

\section{ABSTRACT}

Objective: Study the tourist product focused on the hotels, the restaurants, and the tourist transportation industries, as important components of the tourist offer in Tabasco, from the tourist perspective in order to disclose the status of their attributes regarding the service, the price and the promotion.

Materials and methods: A Quantitative, crosssectional, and descriptive research of the tourist product in Tabasco was done. Data were collected from the National Institute of Statistics, Geography and Informatics, and the Ministry of Tourism, including important contributions from tourist economy authors. 271 questionnaires were applied to tourists, based on the sample population with a confidence level of $90 \%$. In this manner, data on the service provided by the hoteliers, the restaurateurs, and the tourist transport sectors were gathered. Three major variables were studied: product, price and promotion

Results: In all three sectors, services are perceived as "good ones» by tourists where the highest percentages (those above 60\%) were observed in the hotel rooms and food and beverage in restaurants. Prices show a behavior perceived as a "good one» in the 3 tourist sectors, clarifying that only in the hotel rooms there is

* Doctor en Ciencias Económicas con Especialidad en Economía Turística y Comercio Internacional. Profesor-Investigador. División Académica de Ciencias Económico Administrativas. Universidad Juárez Autónoma de Tabasco (UJAT).

** Doctor en Finanzas Públicas. Profesor-Investigador. División Académica de Ciencias Económico Administrativas. Universidad Juárez Autónoma de Tabasco (UJAT).

*** Doctor en Economía. Profesora-Investigadora. División Académica de Ciencias Económico Administrativas. Universidad Juárez Autónoma de Tabasco (UJAT).

Fecha de recibido: 28 de marzo de 2012 Fecha de aceptación: 18 abril de 2012. 
en las promociones; no obstante, los tres sectores son percibidos como bueno, únicamente los restaurantes rebasan el $60 \%$.

Conclusión. Los tres sectores son bien percibidos por el consumidor turístico, en lo que se refiere al servicio, precio, y promoción. Sin embargo, es necesario poner atención al transporte turístico, a pesar de ser percibido como bueno por el turista, sus porcentajes están por debajo del porcentaje de los otros sectores en las tres variables estudiadas. Por lo tanto, los prestadores de servicios deben innovar, especialmente en la transportación turística, para poder estar acorde a los cambios del mercado turístico que evoluciona día a día. a percentage above $60 \%$. Nevertheles, in promotions, even the three sectors are perceived as "good ones», only the restaurants exceed $60 \%$.

Conclusion: The three sectors are well perceived by the tourist consumer in regard to service, price, and promotion. However, it is necessary to pay attention on the tourist transportation, because even though it was perceived as a "good one» by the tourist, its percentages are below the average compared to the other studied variables. Therefore, service providers must make innovations especially in the tourist transportation area, in order to be consistent with the changing tourist market that is evolving day by day.
Palabras clave: Percepción del turista. Producto turístico. Turismo en Tabasco. Consumidor turístico. Turista en Tabasco.
Key words: Tourist Perception. Tourist Product. Tourism in Tabasco. Tourist consumer. Tourist in Tabasco.

DIRECCIÓN PARA RECIBIR CORRESPONDENCIA: Correo electrónico: andresguz18@hotmail.com

e acuerdo a la teoría económica turística, el producto turístico es un bien complejo que se distingue de los otros productos desde el punto de vista que presenta múltiples dimensiones. Es en efecto, una combinación de bienes y servicios que son ofertados por los diferentes sectores, tales como: el transporte, el hospedaje, el restaurantero, la diversión, los espectáculos, el deporte, y las empresas de viajes que buscan satisfacer las necesidades y deseos de los turistas (Boullon, 1997; Caccomo \& Solonandrasana, 2001; Mclntosh R., Goeldner, C. \& Ritchie J., 2001). Estos mismos autores definen a la oferta turística como el conjunto de productos turísticos y de servicios puesto a disposición del usuario turístico por una destinación. Aclarando que, el turista consume uno a uno los componentes de una estancia turística en donde la utilidad global del producto es difícilmente sentida de manera directa y el consumo que es determinado por su duración, es un consumo secuencial (Caccomo \& Solonandrasana, 2001).

Según Cárdenas-Tabares (1986), el producto turístico "está conformado por el conjunto de bienes y servicios que se ofrecen al mercado para un confort material o espiritual en forma individual o en una gama amplia de combinaciones resultantes de las necesidades, requerimientos o deseos de un consumidor al que Ilamamos turista». Esto implica que el producto turístico como bien compuesto (Caccomo \& Solonandrasana, 2001) está conformado por: atractivos naturales, artificiales y los humanos; las facilidades que implica el alojamiento en todas sus formas; la industria de alimentos y bebidas; al entretenimiento y diversión; las agencias de viajes; las arrendadoras de automóviles, y en forma especial, el personal capacitado disponible para atender adecuadamente a los turistas. Pero además, el producto implica que los atractivos sean accesibles, ya sea por barcos, avión, autobús o automóvil (Cárdenas-Tabares, 1986).

Existen destinos turísticos de reputación a nivel mundial, de los cuales se pueden mencionar como ejemplos: Cancún en México, Niza en Francia, Niágara en Canadá, en donde se observa una elevada frecuentación turística. Estos resultados no son obra de la casualidad; la preparación de la oferta turística es determinante en la atracción de los turistas, en donde el servicio soporte sin duda alguna juega un rol crucial. En otras palabras, los componentes tales como la rama hotelera, restaurantera y el transporte turístico, son determinantes en la utilidad global del producto turístico.

Dado que el sector turístico es considerado por la comunidad científica como el más grande a nivel mundial (Mclntosh, et al. 2001) y también uno de los que más empleo genera, no debemos extrañarnos, al observar año tras año a miles de turistas preparando sus vacaciones en función de la oferta turística disponible 
en cada destinación. Esta es definida como «el área geográfica que esta en la medida de ofrecer un producto turístico; es decir, un conjunto de servicios soportes que gravitan alrededor de actividades o experiencias inhabituales por el turista» (Jensen, Hansen \& Mets, 1993 in Framke, 2002). El destino puede estar simbolizado por una sola organización. El turista se encuentra entonces en contacto con un solo proveedor de servicios durante su estancia; sin embargo, los destinos en su mayoría están compuesto por productos y servicios de muchos de los operadores públicos y privados (World Tourism Organization, 1997).

La ausencia o escasa visita a un destino turístico, está estrechamente relacionada con la falta de productos que satisfagan las expectativas de los consumidores del bien o servicio (Kotler, 2002). Si un destino no cuenta con los productos que sean de la preferencia de los usuarios-turistas, en vano serán los esfuerzos invertidos en promoción y comercialización. De hecho, la preparación de la oferta turística requiere de enormes inversiones que el gobierno y los empresarios deben realizar asumiendo los riesgos y costos (Wandner \& Van Erden, 1980 en Fong-Lin, 2003).

No obstante, que algunas destinaciones turísticas hayan sido privilegiadas por sus atracciones y localizaciones, la falta de un servicio soporte adecuado puede afectar la decisión del turista en su elección del lugar donde pasar sus vacaciones. No debemos omitir el concepto de percepción que el turista tiene sobre el producto turístico. De acuerdo a la Real Academia Española, consiste en una sensación interior que resulta de una impresión material hecha en nuestros sentidos. De esta manera el turista puede hacer la diferencia entre las experiencias relacionadas a las atracciones turísticas (Peak Touristic Experiences) y aquellas que sirven de apoyo a esas atracciones o atractivos turísticos, como las ramas restaurantera, hotelera y transporte turístico (supporting Consumer Experiences) (Solima, 2005), dicho de otra manera las (actividades primarias) y las (actividades de soporte). Además, es capaz de evaluar en base a su percepción, que nivel de servicio es el que está consumiendo. Es necesario mencionar que el turista tiene una imagen mental de los destinos, determinada por un cúmulo de datos que le ayudan a hacer una elección del destino de su preferencia.

Así, el conocimiento de la percepción del turista sobre la actividad turística, genera información de gran valor, indispensable para gestión del sector. Como se ha mencionado de manera reiterada se constituye en un negocio compuesto de un gran numero de servicios y empresas competitivas en el transporte, hospedaje, el servicio de alimentos y bebidas, entre otros, en donde los actores del servicio soporte son por lo tanto profesionales autorizados a enmarcar la actividad como los empresarios hoteleros y restauranteros (Lickorish, Jefferson, Bodlender, \& Jenkins, 1994).

El Estado de Tabasco ${ }^{1} y$ sus atractivos, como destino turístico, no están exento de la problemática de baja frecuentación de turistas, los que llegan a Tabasco, casi siempre están de paso y esto puede ser debido a la falta de una oferta interesante que atraiga y retenga al turista por más tiempo, ya que su estancia promedio es muy corta ( 1.6 días en los turistas nacionales y 1.4 días en los turistas internacionales) (Guzmán-Sala, 2010). Esta situación demanda una correcta planeación en los componentes de la oferta turística que permita contar con las características acorde a las preferencias de los turistas-consumidores, logrando que se interesen por esta destinación.

El presente artículo tiene por objetivo, el estudio del producto turístico enfocado hacia los establecimientos hoteleros, restauranteros, y transporte turístico, como componentes importantes de la oferta turística, en Tabasco, desde la perspectiva del turista, con la finalidad de revelar el estado de sus atributos, respecto al servicio, precio y promoción. Es necesario conocer cual es la percepción de los turistas hacia el servicio soporte en la oferta turística del Estado de Tabasco, de acuerdo a Solima (2005): (supporting Consumer Experiences).

\section{MATERIALES Y MÉTODOS.}

La investigación es de corte cuantitativo, transeccional, descriptivo. Los datos recopilados se obtuvieron del: Instituto Nacional de Estadística y Geografía; la Secretaría de Turismo, y algunos artículos provenientes de diversas fuentes. En base al inventario de establecimientos, componentes de los servicios soportes, y la frecuentación turística anual, se determinó el tamaño de la población en estudio, para proceder al cálculo de la muestra con un nivel de confianza del $90 \%$. La población en estudio se determinó tomando en consideración la frecuentación de turistas promedio mensual durante un periodo 3 meses en que se llevó el estudio, obteniéndose una población de 248, 952 turistas. Por lo tanto, en base a la muestra poblacional

\footnotetext{
${ }^{1}$ El Estado de Tabasco se localiza en el sureste de la República Mexicana, en el Golfo de México.
} 
obtenida, se aplicaron 271 cuestionarios a turistas que se encontraban en Tabasco, y que estaban interactuando con los actores del sector, es decir con el servicio soporte (hotelero, restaurantero, transporte turístico). En la formulación de las preguntas se consideraron tres variables importantes: producto, precio y promoción.

Fue necesario hacer una revisión literaria sobre turismo internacional y la contribución de importantes autores nos permitió aproximarnos al conocimiento de la situación real del servicio soporte en Tabasco, cuya problemática no es ajena a lo que sucede con otros destinos turísticos. En donde la situación de la oferta turística, en lo particular los prestadores del servicio de hospedaje, comida y transporte que son considerados el servicio soporte del turismo, también influyen en la decisión del turista al momento de elegir un destino turístico.

\section{RESULTADOS.}

\section{Los servicios en los hoteles, restaurantes y} transporte turístico.

Los resultados del estudio muestran que los turistas en su mayoría consideran bueno el servicio brindado en la habitación de hotel con $66 \%$, además de un $16 \%$ que lo considera excelente. Sin embargo, un 15\% lo percibe como regular, $2 \%$ malo y $1 \%$ pésimo. En términos generales el servicio que se ofrece en los

FIGURA 1. PERCEPCIÓN SOBRE EL SERVICIO.

\section{Producto turístico}

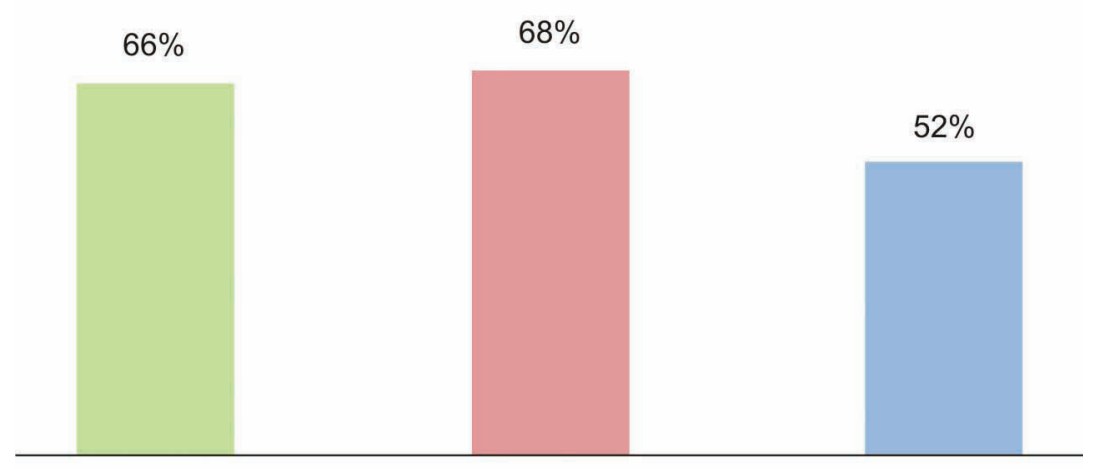

Producto habitación

Producto restaurante

Producto transporte

Fuente: Elaboración propia. Tendencia porcentual del servicio percibido como bueno, en los subsectores: hotelero, restaurantero, y transporte. hoteles de Tabasco, según la percepción del turista es bueno ya que más del $60 \%$ de los turistas entrevistados así lo consideran (ver figura 1). En los restaurantes el servicio es bueno un $68 \%$ y excelente con el $18 \%$. Pero un grupo importante lo considera regular, $13 \%$. Esto significa que el servicio ofertado por parte de la industria restaurantera es bueno, y cumple con las expectativas esperadas por el consumidor-turista. Además, en el transporte que es un complemento para el adecuado servicio turístico, se observa que el turista considera que el servicio es bueno en un $52 \%$, excelente en un $15 \%$, regular en un $27 \%$, malo $5 \%$, lo que muestra que el transporte turístico brindado es bueno, dado que la mayoría de los turistas así lo consideran, pero además observamos un porcentaje interesante de turistas que afirmaron que el servicio de transporte es excelente.

Los resultados sobre el servicio en los tres sectores muestran que es percibido como bueno, en donde los porcentajes más elevados por arriba del $60 \%$ fueron encontrados como buenos en habitaciones de hotel y comida. Sin embargo, se nota una tendencia distinta en el transporte turístico que está debajo del $60 \%$. En este subsector, no obstante que el porcentaje mayor se ubique en bueno, apenas alcanza el $50 \%$, pero además un porcentaje importante $27 \%$, considera al transporte como regular.

\section{Los precios en los servicios soportes.}

Por lo que respecta a los precios en las habitaciones de hotel, los turistas consideran que el precio es bueno $65 \%$, regular $20 \%$, excelente $12 \%$ y malo en $2 \%$. Como se puede observar, los turistas consideran que el precio en las habitaciones de los hoteles en el Estado es bueno; en la industria restaurantera, el precio percibido esta en el gusto de los consumidores-turistas de la siguiente manera: bueno $59 \%$, regular $21 \%$, excelente $18 \%$, malo en $2 \%$, lo cual quiere decir que los precios son interesantes en este subsector. $Y$ en el transporte turístico, los precios son percibidos como bueno $58 \%$, regular con $28 \%$, excelente con $11 \%$, y malo al registrar 3\%. De acuerdo a estos porcentajes el precio es bueno en general y sobre todo si tomamos en consideración los resultados de un porcentaje interesante que afirman que el precio es excelente. 
FIGURA 2. PERCEPCIÓN SOBRE EL PRECIO EN LOS SERVICIOS SOPORTES.

\section{Precio del producto turístico}

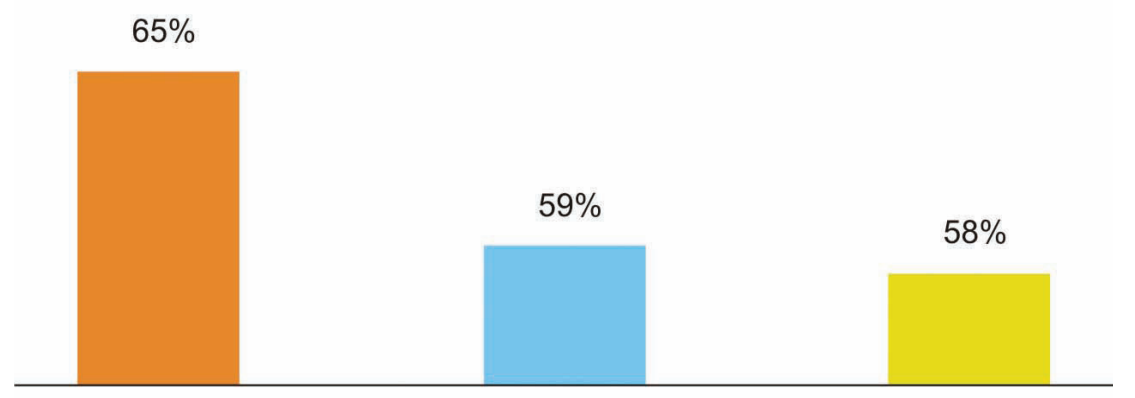

Producto habitación

Producto restaurante

Producto transporte

Fuente: Elaboración propia. Tendencia porcentual del precio percibido como bueno, en los subsectores: hotelero, restaurantero y transporte.

FIGURA 3. PERCEPCIÓN SOBRE LA PROMOCIÓN.

\section{Promoción producto turístico}



Fuente: Elaboración propia. Tendencia porcentual de las actividades promocionales percibida como bueno, en los subsetores: hotelero, restaurantero y transporte.

Podemos concluir con los resultados de la investigación que los precios en los 3 sectores turísticos son igualmente percibidos como bueno, pero solo el servicio de habitación de hotel está por arriba del $60 \%$. A pesar de todo, el precio está en el gusto del consumidor turista más allá de especulaciones sobre precios elevados debido a la actividad petrolera. Aunque son porcentajes calificados como buenos, tanto restaurantes como transporte turístico, se observan porcentajes por debajo del 60\% (ver figura 2).
Las promociones en los hoteles, restaurantes y transporte.

En las promociones de los hoteles, se obtuvieron los siguientes datos: regular $24 \%$, bueno $58 \%$, excelente $13 \%$, y malo $4 \%$. Esto nos da evidencias de que si se realizan promociones en los hoteles, y consideradas como buenas de acuerdo a las respuestas del consumidor turístico. En el caso de los restaurantes el resultado respecto a las promociones fue el siguiente: bueno $62 \%$, regular $26 \%$, excelente $10 \%$. pésimo $1 \%$, y malo $1 \%$. Sin duda las promociones en los restaurantes son buenas; por ultimo, no dejando de ser importante, el transporte turístico en promociones se observa en bueno $56 \%$, regular $33 \%$, excelente $7 \%$, malo $3 \%$, y pésimo con $1 \%$.

Puede observarse que en los tres sectores se mantiene como bueno en las promociones, pero únicamente los restaurantes logran superar el $60 \%$, mientras que los otros dos sectores están debajo del $60 \%$. Esto indica la necesidad de un esfuerzo relativo por el uso de las promociones para capturar una mayor preferencia en el consumidor en los hoteles y transporte turístico (ver figura 3).

\section{DISCUSIÓN.}

La oferta turística es el elemento que dinamiza una destinación. Cada destinación por medio del intermediario de la organización que la administra debe poner al turista en el centro de sus decisiones (Duncan, 2002). Pero ¿podrá el intermediario lograr este objetivo cuando la destinación no cuenta con una oferta atractiva a la vista del usuario-turista? Definitivamente no. En hechos es crucial para cualquier destino turístico una adecuada preparación y desarrollo de la oferta turística (Caccomo \& Solonandrasana, 2001); el Estado de Tabasco como tal, debe considerar estas acciones con el fin de atraer al turista doméstico, pero principalmente al turista internacional cuyo índice de frecuentación es relativamente bajo en esta entidad (Guzmán-Sala, 2010). 
De acuerdo con Mclntosh etal. (2001), la oferta turística está compuesta de varios componentes en el que destacan los subsectores hoteleros, restaurantero, y transporte. Así, el servicio soporte en la oferta turística de Tabasco está compuesto de aproximadamente 1,000 establecimientos turísticos, que operan en varias ramas de la actividad tales como: restaurantes, hoteles y transporte turístico. Asimismo, es absolutamente indispensable que estos establecimientos presten servicios interesantes a la vista del turista.

Por lo tanto, las interrogantes que deben responderse son, ¿Cómo percibe estos tipos de servicios, el turista que llega a Tabasco? ¿Son realmente interesantes los precios y las promociones en el servicio soporte de Tabasco?

De acuerdo con los resultados de la investigación, el producto turístico en los tres sectores es percibido como bueno, sobre todo en habitaciones de hotel y la comida en restaurantes. Sin embargo, en el transporte turístico se observa una tendencia distinta en disminución. Bien que el porcentaje mayor se ubique en «bueno», apenas logra el $52 \%$, pero además un porcentaje importante $27 \%$ considera al transporte como «regular». Podemos deducir que aunque el turista considera bueno el transporte turístico, es posible que hayan ciertas deficiencias que se necesiten mejorar, el transporte es un componente clave en el desarrollo de un destino turístico en donde el confort, rapidez y seguridad, además del precio, son aspectos a considerar al momento de estructurar la oferta turística (De la Torre, 2000).

Por otra parte, los precios son en cierta medida determinante en la decisión del turista para visitar una destinación, el turista generalmente elige la destinación menos costosa. A la luz de la investigación, puede decirse que los precios en los 3 sectores turísticos, estudiados en Tabasco, son bien percibidos. El precio está en el gusto del turista; pero llama la atención el hecho de que en la habitación de hotel los porcentajes considerados como buenos superan el $60 \%$, mientras que en restaurante y transporte se observa una tendencia debajo del $60 \%$. Este resultado es importante dado que existen especulaciones que el servicio de hotel es más caro en Tabasco que en otras entidades, dato que se profundiza abordando este mismo estudio en otras entidades y haciendo un análisis comparativo de los precios, en donde seguramente habrán conclusiones más concretas. Además, el porcentaje más bajo aunque considerado como bueno se encuentra en el transporte con $58 \%$.

Finalmente, en el apartado de las promociones que tienen por objetivo mantener la fidelidad del consumidor, atraer nuevos consumidores y/o dar a conocer un nuevo producto (Kotler, 2002), los tres sectores mantienen la misma tendencia en lo que a promociones se refiere, considerados como bueno, pero solo en restaurant se observa un porcentaje mayor al $60 \%$; por el lado del transporte turístico una vez más obtuvo el porcentaje menor. Sin duda alguna, muestra que el uso de las promociones para capturar una mayor preferencia en el consumidor es necesario, pero solo los restaurantes están explotando ésta herramienta de marketing. Es necesario que los empresarios hoteleros y transporte turístico, reflexionen sobre las ventajas de las promociones, pero será interesante que estos realicen ciertas innovaciones para obtener mejores beneficios.

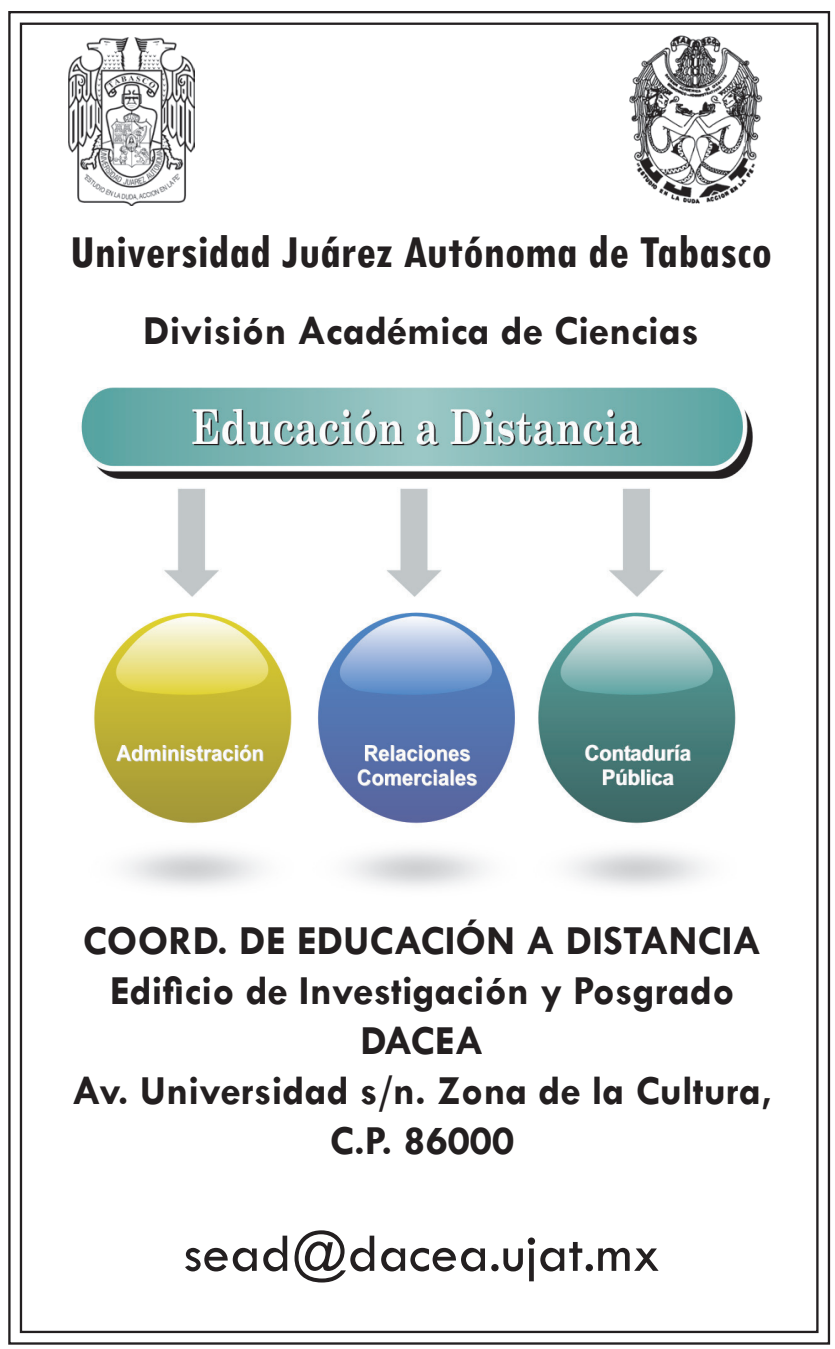

HITOS DE CIENCIAS ECONÓMICO ADMINISTRATIVAS 


\section{CONCLUSIÓN.}

La oferta turística del Estado de Tabasco, en lo que concierne a los servicios que sirven de soporte al sector y que está integrada por los establecimientos hoteleros, restauranteros y transporte turístico (Solima, 2005), es percibida por los turistas como buena. En efecto, los tres sectores son bien percibidos por el consumidor turístico en las variables que se estudiaron: servicio de habitación, precio y promoción. Es necesario poner atención al hecho que el transporte turístico tenga los porcentajes más bajos en el estudio, aunque sea percibido como bueno por el usuario. Si se profundiza en el estudio incluyendo otras variables, es posible que existan ciertas deficiencias que lo hacen vulnerable ante los otros subsectores.

Los prestadores de servicios deben estar conscientes que el consumidor turístico evoluciona día a día; por lo tanto, innovar es una acción obligatoria para poder competir en un mercado competitivo, en donde por la misma naturaleza del producto al presentar altos grados de complejidad se necesita tener bien estructurada la oferta (Caccomo \& Solonandrasana, 2001) en las actividades primarias y en el servicio soporte. Las destinaciones podrán contar con una frecuentación elevada en la medida que los actores del sistema turístico estén a la altura de las expectativas del turista nacional e internacional. El objetivo es claro para Tabasco como destino turístico, si desea poner al turista en el centro de sus decisiones (Duncan, 2002), antes que cualquier otra acción, se necesita contar con una oferta atractiva en donde el servicio proporcionado por los prestatarios hoteleros, restauranteros y transporte de turistas, estén a la altura de las exigencias del turista. No debe pasar desapercibido que el turista puede hacer la diferencia entre las actividades primarias y las de soporte al momento de consumir el producto turístico.

\section{REFERENCIAS}

Archer, B. (1977). Tourism Multiplieres. The State of the Art Cardiff: University of Wales.

Boullon, R. C. (1997). Planificación del Espacio Turístico (3rd Edición). México: Ed. Trillas.

Caccomo , J. L., \& Solonandrasana, B. (2001). Innovations dans l'industrie touristique. París: Harmattan.

Cárdenas-Tabares, F. (1986). Producto Turistico. Trillas Turismo.

Crompton, J L. (1979). Motivations for pleasure vacation. Annals of Tourism Research 6 (4) 408-424.

De la Torre, F. (2000). Sistema de Transportación Turística. México: Trillas.

Duncan, T. (2002). Using Advertising and Promotion to build brands. McGraw-Hill.

Fong-Lin, C. (2003). Forecasting tourism demand: a cubic polynomial approach. Tourism Management, 209-218.

Framke, W. (2002). The Destination as a Concept: A Discussion of the Business-related Perspective versus the Socio-cultural Approach in Tourism Theory. Scandinavian Journal of Hospitality and Tourism, 2, 2, 93-108.
Guzmán-Sala, A. (2010). Le Secteur Touristique du Tabasco au Mexique: Analyse Opérationnelle et Perspective en Termes d'Offre et Demande Touristiques. Sarrebruck, Alemania: Éditions Universitaires Éuropéenes.

Instituto Nacional de Estadística Geografía e Informática. (2010). Anuario estadistico del estado de Tabasco. Villahermosa, Tabasco.

Kotler, P. (2002). Dirección de Marketing Conceptos Esenciales. Pearson.

Lickorish, L. J., Jefferson, A., Bodlender, J., \& Jenkins, C. L. (1994). Desarrollo de destinos turísticos. México D.F.: Diana.

McIntosh R., Goeldner, C. \& Ritchie J. (2001). Turismo: Planeacion, Administracion y Perpspectiva. Mexico: Limusa Wiley.

Solima, L. (2005). De la Valeur du territoire aux instruments d'intégration de la tarification, dans la culture mise á prix. . L'Harmattan.

World Tourism Organization. (1997). International Tourism: A global Perspective. Madrid, Spain: WTO. 